\title{
The hydration problem in solution biophysics: an introduction
}

\author{
Stephen E. Harding \\ NCMH Physical Biochemistry Laboratory, School of Biosciences, University of Nottingham, Sutton Bonington LE12 5RD, \\ $U K$
}

Received 14 August 2001

\begin{abstract}
The background and purpose of the British Biophysical Society Discussion meeting, The Hydration Problem in Solution Biophysics, held at the University of Glasgow, 12 September 2000, is described, particularly in relation to previous meetings in this field. Whereas a study of the nature and dynamic properties of water associated with a molecule is an important topic by itself, the collection of papers based on this meeting focus mainly on its affect in interpreting biophysical data in terms of macromolecular shape in a solution environment, particularly under dilute and very dilute systems. The techniques considered are largely hydrodynamically or thermodynamically based and supplemented by molecular modeling strategies; but in the context of how these could be used in conjunction with techniques like X-ray crystallography, NMR and neutron scattering. ( 2001 Elsevier Science B.V. All rights reserved.
\end{abstract}

Keywords: Hydration problem; Solution biophysics; Dilute systems

\section{Introduction}

The papers in this special edition of Biophysical Chemistry derive from the 2001 British Biophysical Society Discussion Meeting held at the University of Glasgow in conjunction with the Biochemical Society. They address a problem which has, for nearly a century, had resonances complicating and obscuring attempts at describing the structure of biological macromolecules in the

E-mail address: steve.harding@nottingham.ac.uk (S.E. E-mail
Harding). environment in which many occur naturally: an aqueous solution. Whereas a study of the nature and dynamic properties of water associated with a molecule is an important topic by itself, the following collection of papers focus mainly on its affect in interpreting biophysical data in terms of macromolecular shape in a solution environment.

\section{What is the hydration problem?}

The tools of X-ray crystallography and nuclear magnetic resonance spectroscopy have provided 
the high-resolution structures for thousands of protein molecules. There is currently significant interest in how both these techniques, together with others such as neutron crystallography can be used to throw light on the nature and dynamics of the water molecules associated with biological macromolecules [1].

There are many biological macromolecules that are, however, beyond the reach of these powerful probes: current restrictions include problems of crystallization and macromolecular flexibility in the case of crystallography, and one of size for NMR, with the limit for routine application of $M_{r} \sim 40000$. In these cases, hydrodynamic and solution scattering probes can be used. Although these have the advantage of being able to probe very dilute solutions of macromolecules and without size limitation, they are of necessity low-resolution, giving the overall shape or topology of a macromolecule. Interpretations of the data can also be obscured because the parameters involved are often equally as sensitive to macromolecular hydration (or we should say time-averaged macromolecular hydration to recognize the fact that it is a dynamic process) as they are to macromolecular shape: this is the hydration problem.

\section{Early attempts at dealing with the hydration problem}

Hydrodynamicists have tried to package the contribution of the water associated with a macromolecule to a measured property such as the sedimentation coefficient, translational or rotational diffusion coefficients, or intrinsic viscosity into a single parameter traditionally given the symbol $w$ [2] or $\delta$ [3], defined in terms of the time-averaged mass of water/solvent associated with a macromolecule per unit anhydrous mass of macromolecule. Historically, for proteins, values between 0.2 and 0.5 have been estimated or assumed for this parameter. For glycosylated proteins and carbohydrate polymers, larger values have been assumed because of the generally higher affinity for water of carbohydrates: assuming $\delta$ values have a high degree of arbitrariness for shape evaluations, a rather unsatisfactory scenario. One of the earliest attempts of addressing this was that of Oncley, who in 1941, [4] proposed a combination of two different types of hydrodynamic measurement so as to provide an estimate of the shape in terms of the axial ratio of the equivalent prolate or oblate ellipsoid of revolution. The hydration contribution could be eliminated by using a graphical method. Further progress was made by Scheraga and Mandelkern in 1953 [5], who proposed combining the sedimentation coefficient and the intrinsic viscosity in an analytical way. By 1983 a host of hydration independent shape functions, for not only ellipsoids of revolution but also general triaxial ellipsoids [6] and bead models, [7] had been generated. However, these combined shape functions came at a price - lower sensitivity to shape. Therefore, rather than trying to shirk away from this problem by using these rather insensitive functions, we can at last begin to meet it head-on, by taking advantage of the inroads that the high resolution and even low resolution probes have been making to predict what the time averaged hydration is for a particular protein, nucleic acid or glycopolymer.

\section{Previous meetings on hydration}

One of the first meetings convened to address the water relations of macromolecules was that held at Vermont USA, October 1965 and organized by J.H. Bland [8]. In that meeting J.D. Bernal was among several speakers who emphasized that water was itself structured and he described the biological implications for this [9]. In a seminal paper, A.G. 'Sandy' Ogston pointed out that specification of hydration merely as a weight or volume of water per weight of solute gives only preliminary information, but nonetheless examined its consequences for thermodynamic and hydrodynamic data [10]. Ogston could even then see the promise of NMR and other spectroscopic methods in being able to discriminate between the specific contributions of water to a particular system. Indeed Ogston throughout his lifetime had made many seminal contributions to the general field of Physical Biochemistry: it is to him this special edition of Biophysical Chemistry is 
dedicated. Ten years later, in 1975 Richards and Franks convened a Royal Society Discussion meeting on Water Structure and Transport in Biology [11], a meeting in which it was clear that researchers were starting to tackle the issue of the dynamics of hydrated water (see, e.g. [12]). After a gap of 21 years a Faraday Discussion Meeting on Hydration Processes in Biological and Macromolecular Systems organized by Professor Finney was convened over three days in April 1996 [13] to assess the substantial progress during those years, particularly with regards to our understanding of the dynamics of the hydration process. In that meeting, Wuthrich et al. [14] had explained how individual hydration water molecules exchange in and out of hydration sites of a macromolecular structure and how nuclear Overhauser enhancement NMR procedures can measure average water residence times. Such residence times were generally very short (10-200 ps) for surface hydration water and generally longer for interior, solvent inaccessible hydration water. These researchers were also able to consider in some detail the hydration dynamics of protein-nucleic acid complexes by using supporting data from molecular dynamic simulations. Denisov and Halle, in a related study using NMR dispersion procedures [15] had considered five globular proteins (ribonuclease A, lysozyme, myoglobin, trypsin and serum albumin) and found from water, oxygen-17 and deuteron spin relaxation rates long lived residence times (in the range $1 \mathrm{~ns}$ to $1 \mathrm{~ms}$ ) for so-called integral water molecules seen in crystal structures. These integral water molecules are either buried in internal cavities, trapped in narrow clefts or coordinated to metal ions: such cavities were shown to be not empty but hydrated. For the surface water layer these researchers found very fast average residence times (10-50 ps) and they also demonstrated entrapment of water between structural domains of large proteins.

\section{Hydrodynamics, thermodynamics and modeling strategies}

We have avoided consideration of those topics addressed at the 1996 meeting [13]. Therefore, for example, neither NMR studies of the hydration dynamics and exchange rates of proteins and nucleic acids in solution nor the potentials of mean force strategy have been considered in detail here (with the exception of carbohydrates). Instead, the one day BBS discussion meeting held at Glasgow focussed mainly on hydrodynamic and thermodynamic procedures, supplemented by molecular modeling strategies, but in the context of how these could be used in conjunction with techniques like X-ray crystallography, NMR and neutron scattering. This follows the demonstration by McBrierty et al. at the 1996 meeting [16] of the virtue of using several techniques to tackle hydration rather than one in isolation. In the first paper, Rowe [17] gives a global view on the concept of hydration of proteins and shows how from DLVO theory we can see that hydration plays a much more critical role than electrostatic repulsive phenomena in the stability of protein solutions: the use of DLVO theory enables us to understand a broad spectrum of the solution properties of proteins, provided that the presence of a hydration layer of approximately $5-6 \AA$ is allowed for. The following paper by Engelsen [18] then turns to carbohydrates and describes how from molecular dynamic simulations in conjunction with NMR measurements of rotational and translational diffusion coefficients of small carbohydrates we can make the conclusion that in terms of the overall hydration, different carbohydrates have similar hydration characteristics, although individual carbohydrate-water interactions play a pivotal role in local conformation. This is followed by a paper by Perkins [19] who, using X-ray and neutron solution scattering studies, claims that for a protein a time-averaged hydration value of $\delta=0.3$ and a water molecule volume of $24.5 \AA^{3}$ are reasonable: for neutron scattering studies it is not necessary to incorporate a hydration shell, as to a good approximation this is not observable. Durschlag and Zipper [20] claim a higher value for $\delta$ of 0.35 using, particularly, X-ray scattering — as long as average properties of proteins are considered. Garcia de la Torre [21] use hydrodynamic bead model approaches and the algorithms HYDROPRO and 
HYDRONMR to obtain average values for $\delta$ of 0.3 and a surface hydration layer of $1.2 \AA$ thickness. Despite noticeable differences in these parameters from one protein to another, he claims that for the main purpose of predicting protein solution properties these values can be safely used. Zhou [22] notes the wide range of predicted hydration values $(\delta=0.3-0.5)$ for medium sized proteins based on X-ray diffraction, NMR, spectroscopy, calorimetry, infrared spectroscopy and molecular dynamics simulation. By extending the hydrodynamic approach using translational and rotational diffusion and intrinsic viscosity properties to incorporate published crystal structures and electrostatic properties (capacitance and polarization), he now claims a unified picture of protein hydration emerges with preferred hydration sites around a protein surface. These sites are occupied nearly all the time, but by different molecules at different times, so that even though a given water molecule may have a short residence time, (e.g. 100 ps from NMR) the site appears fully occupied when methods based on time-averaged properties are used.

Carrasco et al. [23] then introduce Crystallohydrodynamics as a means of evaluating the low resolution structures of multi-domain molecules in terms of orientation of domains. Hydration is dealt with by combining crystal structures for the individual domains with the measured solution properties of these domains: for glycoproteins like $\mathrm{IgG}$ antibody classes a value for $\delta$ of $\sim 0.5$ is found for the Fab' domain and $\sim 0.7$ for the glycosylated $\mathrm{Fc}$ domain. A weight average value for the intact structure is then taken which, when combined with measured solution properties from that provides the low resolution domain orientation, and giving the correct prediction for a hingeless mutant antibody whose structure is known.

Allison [24] then presents a complementary approach to bead modeling using boundary element methodology for modeling a range of transport phenomena in dilute solution. Modeling is carried out by representing the biomolecule as an arbitrary array of solid platelets that contains fixed charges within, and with the surrounding fluid, represented as an electrodynamic and hydrodynamic continuum, allowing for ion relaxation. For example, applied to a 20-base-pair DNA it is found to be unnecessary to include a layer of bound water to reconcile experimental and model translational diffusion coefficients.

The theme then turns to thermodynamics with two papers. The first is a paper by Cooper et al. [25], who describe how that, since modern microcalorimetry includes accurate measurement of heat changes of macromolecule in aqueous solution, these changes can reflect changes in hydration under these conditions. These researchers also describe the new technique of Pressure Perturbation Calorimetry and indicate its enormous potential for studying hydration/solvation-related volumetric changes in biomolecules at modest pressure. The second thermodynamic paper and final paper of this collection [26] is by Winzor et al., who analyze thermodynamic non-ideality in terms of protein hydration by correlating measured second thermodynamic virial coefficients (from, for example, sedimentation equilibrium in the analytical ultracentrifuge, osmotic pressure or light scattering experiments) with polyelectrolyte and exclusion volume properties, the latter of which depends on $\delta$. The dependence of the effective solvated radius upon protein molecular mass exhibits reasonable agreement with the relationship calculated for a model, in which the anhydrous protein molecule is surrounded by a 5.2- $\AA$ hydration shell. Statistical mechanical interpretation of the magnitudes of second virial coefficients in terms of the size of the hydrated particle leads to a magnitude of the hydration parameter that is larger than the usually assumed value of 0.3 for $\delta$ - the concept of an invariant $\delta$ with molecular mass also becomes open to question.

Thus, what progress has been made in our ability to tackle the hydration issue clouding macromolecular shape or surface topology measurements in highly dilute systems? The following papers in this special edition will now examine this question in detail.

\section{References}

[1] J.L. Finney, Overview lecture. Hydration processes in biological and macromolecular systems, Faraday Discuss. Chem. Soc. 103 (1996) 1-18. 
[2] P.G. Squire, M. Himmel, Hydrodynamics and protein hydration, Arch. Biochem. Biophys. 196 (1979) 165-177.

[3] C. Tanford, Physical Chemistry of Macromolecules, John Wiley and Sons, New York, 1961.

[4] J.L. Oncley, Evidence from physical chemistry regarding the size and shape of protein molecules from ultracentrifugation, diffusion, viscosity, dielectric dispersion and double refraction flow. Ann. N.Y. Acad. Sci. 41 (1941) $121-150$

[5] H.A. Scheraga, L. Mandelkern, Consideration of hydrodynamic properties of proteins, J. Am. Chem. Soc. 75 (1953) 179-184.

[6] S.E. Harding, J.C. Horton, H. Colfen, The ELLIPS suite of macromolecular conformation algorithms, Eur. Biophys. J. 25 (1997) 347-359.

[7] J. Garcia de la Torre, B. Carrasco, S.E. Harding, Eur. Biophys. J. 25 (1997) 361-372.

[8] J.H. Bland, Water: water binding; structure: hydration and solution theory, Fed. Proc. 25 (1966) 951-2002.

[9] J.D. Bernal, The structure of water and its biological implications. Symposia of the Society for Experimental Biology 19 (1963) 17-32.

[10] A.G. Ogston, Macromolecular hydration in solution, Fed. Proc. 25 (1966) 1993.

[11] R.E. Richards, F. Franks (Eds), Phil. Trans. Roy. Soc. Lond. B, vol. 278, 1977

[12] K.J. Packer, The dynamics of water in heterogeneous systems. Phil. Trans. Roy. Soc. Lond. B 278 (1977) 59.

[13] J.L. Finney, Hydration processes in biological and macromolecular systems, Faraday Discuss. 103 (1996) $1-395$.

[14] K. Wüthrich, M. Billeter, P. Güntert, P. Luginbühl, R. Riek, G. Wider, NMR studies of the hydration of biological macromolecules, Faraday Discuss. 103 (1996) $245-253$.

[15] V.P. Denisov, B. Halle., Protein hydration dynamics in aqueous solution, Faraday Discuss. 103 (1996) 227-244.

[16] V.J. McBrierty, C.M. Keely, F.M. Coyle, H. Xu, J.K. Vij, Hydration and plasticization effects in cellulose acetate: Molecular motion and relaxation, Faraday Discuss. 103 (1996) 255-268.
[17] A.J. Rowe, Probing hydration and stability of protein solutions - a colloid science approach. Biophys. Chem. 93 (2001) 93-101.

[18] S.B. Engelsen, The diluted aqueous solvation of carbohydrates as inferred from molecular dynamics simulations and NMR spectroscopy. Biophys. Chem. 93 (2001) 103-127.

[19] S.J. Perkins, X-ray and neutron scattering analyses of hydration shells: a molecular interpretation based on sequence predictions and modeling fits. Biophys. Chem. 93 (2001) 129-139.

[20] H. Durchslag, P. Zipper, Comparative investigations of biopolymer hydration by physicochemical and modeling techniques. Biophys. Chem. 93 (2001) 141-157.

[21] J. Garcia de la Torre, Hydration from hydrodynamics. General considerations and applications of bead modeling to globular proteins. Biophys. Chem. 93 (2001) 159-170.

[22] H.-X. Zhou. A unified picture of protein hydration. Prediction of hydrodynamic properties from known structures. Biophys. Chem. 93 (2001) 171-179.

[23] B. Carrasco, J. Garcia de la Torre, K.G. Davis, S. Jones, D. Athwal, C. Walters, D.R. Burton, S.E. Harding, Crystallohydrodynamics for solving the hydration problem for multi-domain proteins: open physiological conformations for human IgG. Biophys. Chem. 93 (2001) 181-196.

[24] S.A. Allison, Boundary element modeling of biomolecular transport. Biophys. Chem. 93 (2001) 197-213.

[25] A. Cooper, C.M. Johnson, J.H. Lakey, M. Nöllmann, Heat does not come in different colors: entropy-enthalpy compensation, free energy windows, quantum confinement, pressure perturbation calorimetry, solvation and the multiple causes of heat capacity effects in biomolecular interactions. Biophys. Chem. 93 (2001) 215-230.

[26] D.J. Winzor, L.E. Carrington, S.E. Harding, Analysis of theromodynamic non-ideality in terms of protein solvation. Biophys. Chem. 93 (2001) 231-240. 